\title{
Caseous lymphadenitis in a goat: a case report
}

\begin{abstract}
Corynebacterium pseudotuberculosis is the causative agent of caseous lymphadenitis (CLA), a common disease in small ruminant populations across the globe. The following report describes two cases of a 3 year old Boer cross does showing large mass beneath the jaw, on the ventral side of the mandible with strong pain reaction to touch of the affected region. The consistency of the mass was non-movable, solid and hard on deep palpation. The mass was lanced and purulent discharges were evacuated. Phenotypic and biochemical identification methods allocated the isolates in C. pseudotuberculosis biovar ovis. The vast majority of the isolates was able to produce phospholipase $\mathrm{D}$ and was susceptible to most of the antimicrobial compounds tested. Furthermore, the isolates were compared with C. pseudotuberculosis isolated from other states with different geographical locations and showed high similarity index, suggesting the prevalence of dominant clones and a potential dissemination across the country.
\end{abstract}

Keyword: Boer cross does; Caseous lymphadenitis; Corynebacterium pseudotuberculosis; Bacterial identification; Abscess 